\title{
Nanotechnology - A Panoramic Outlook of its Applications and Future in Dentistry
}

\author{
Amina $^{1}$, Saad Ahmed ${ }^{2}$, Geeta Rajput ${ }^{3}$, Sabzar Abdullah ${ }^{4}$ \\ ${ }^{1}$ Assistant Professor, Department of Prosthodontics, Crown \& Bridge, Dr Ziauddin Ahmad Dental College and \\ Hospital, Aligarh Muslim University, Aligarh \\ ${ }^{2}$ Associate Professor, Department of Oral Pathology \& Microbiology, Rama Dental College. \\ ${ }^{3}$ Professor, Department of Prosthodontics, Crown \& Bridge, Dr Ziauddin Ahmad Dental College and Hospital \\ Aligarh Muslim University \\ ${ }^{4}$ Assistant Professor, Department of Prosthodontics, Crown \& Bridge, Dr Ziauddin Ahmad Dental College and \\ Hospital, Aligarh Muslim University \\ Corresponding Author: Amina
}

DOI: https://doi.org/10.52403/ijhsr.20220224

\begin{abstract}
Nanotechnology has been around since the beginning of time but its emergence in the field of science and technology began in the late 1950's since then it has grown leaps and bound in different fields of science including dentistry. This paper aims to highlight and review the advent, current scenario and the future of nanodentistry which is an integrated terminology formulated by the combination of nanotechnology and dentistry.
\end{abstract}

Keywords: Nanodentistry, Nanotechnology, Dentistry

\section{INTRODUCTION}

Word nano is derived from Greek word "Nanos" meaning one billionth $\left(10^{-9}\right)$ of a particle. The term "nanotechnology" was coined by Prof. Eric Drexler. Nanotechnology was discovered by American physicist Dr Richard Phillips Feynman in December 1959 in his paper "There are plenty of rooms at the bottom".

According to National Nanotechnology Initiative, "Nanotechnology is the understanding and control of matter at the dimensions of roughly 1-100 $\mathrm{nm}$, where unique phenomena enable novel applications. Encompassing nanoscale science, engineering and technology, nanotechnology involves imaging, measuring, modelling and manipulating matter at this length scale".

Nanotechnology is advantageous in dentistry because at the nanoscale level, the chemical, physical, and biological properties of materials differ in valuable and fundamental ways from the properties of individual atoms and molecules or bulk matter.

The power of nanotechnology lies in its potential to revolutionize and transform multiple technology and industry sectors, including agriculture, aerospace, biotechnology, medicine, homeland security and national defense, environmental improvement, information technology, energy, and transportation.

Along with benefits nanotechnology has certain risk factors due to greater difference between the surface area and unit weight, and high surface reactivity of nanoparticles which might lead to greater absorption through skin, lungs, and digestive tract $^{2}$. Tools for applying nanotechnology includes atomic force microscope and scanning tunnelling microscope. 


\section{APPLICATIONS OF NANODENTISTRY}

There are 4 main approaches for application of nanotechnology: Bottom up approach, Top down Approach, Functional Approach, Biomimetic Approach.

Bottom up approach arranges smaller components into more complex assemblies, having extremely strong covalent bonds ${ }^{2}$.

Top down approach uses larger devices to produce smaller ones having precision in assembly and structure ${ }^{1}$.

Functional approach develops components of desired functionality not taking into consideration how they might be assembled ${ }^{3}$.

Biomimetic approach applies
biomolecules for application in
nanotechnology ${ }^{3}$.

\section{BOTTOM UP APPROACH}

NANOROBOT: A nanorobot is a specialized nanomachine, typically 0.5 to 3 microns large with 1-100 nm parts made of chemically inert forms of carbon. Its main applications in dentisty includes Induction of local anaesthesia, hypersensitivity cure, dentrifices (dentifrobots) and major tooth repair.

NANOANESTHESIA: Nanotechnology uses millions of active analgesic nanometer sized dental nanorobots for LA. After contacting the surface of crown or mucosa, the ambulating nanorobots reach the pulp via tubules and gingival sulcus. On reaching the dentin, the nanorobots, within 100 seconds, are said to enter dentinal tubules. Once instilled in the pulp, dental nanorobots may be commanded by the dentist to shut down all sensitivity in any particular tooth that requires treatment. After oral procedures are completed, the dentist orders the nanorobots to restore all sensation and to egress from the tooth by similar pathways used for ingress ${ }^{4}$.

HYPERSENSITIVITY CURE: Dentin hypersensitivity may be caused by changes in pressure transmitted hydrodynamically to the pulp. Hypersensitive teeth have eight times higher surface density of dentinal tubules and tubules with diameters twice as large as non sensitive teeth.Reconstructive dental nanorobots selectively and precisely occlude selected tubules in minutes, thus treating hypersensitivity ${ }^{4}$.

\section{DENTAL DURABILITY AND COSMETICS:}

Covalently bonded artificial materials such as, sapphire or diamond in a fracture resistant nanostructured composite material that include carbon nanotubes are used for replacing upper enamel layers for aesthetic purposes $^{5}$.

DENTIFROBOTS: Nanorobotic dentifrice (dentifrobots) delivered by mouthwash or toothpaste patrol all supragingival and subgingival surfaces at least once a day. Metabolizes trapped organic matter into harmless and odorless vapours, perform continuous calculus debridement and identifying and destroying pathogenic bacteria residing in the plaque and elsewhere. Allows around 500 species of harmless oral microflora to flourish in a healthy ecosystem ${ }^{4}$.

\section{ORTHODONTIC NANOROBOTS:}

Orthodontic nanorobots could directly manipulate the periodontal tissues including gingiva, periodontal ligament, cementum and alveolar bone. Allowing rapid painless tooth straightening, rotating, and vertical repositioning in minutes to hours ${ }^{6}$.

CANCER DIAGNOSIS: Nanotechnology may permit less invasive, less uncomfortable means of identifying and quantifying the markers of disease. Aids in cancer diagnosis, monitoring recurrence or metastasis, and defining the locations, biologic types, and behaviour of malignancies ${ }^{4}$. 


\section{THERAPEUTIC}

NANOTECHNOLOGY: Provide the ability to co-localize delivery of multiple and complimentary therapeutic agents. Materials that now require injection potentially could be inhaled or swallowed .Vaccines may be made more comfortable, by using multitudes of micro- or nanometerscale needles. Nanoparticulates also may allow increases in the amount of drug that reaches abnormal cells. It also has the ability to cloak off potentially toxic therapies ${ }^{7}$.

\section{TISSUE ENGINEERING AND DENTISTRY:}

Tissue engineering has applications in treatment of orofacial fractures, bone augmentation, cartilage regeneration of TMJ, pulp repair, PDL regeneration and implant osseointegration. Range of dimension of nano structures is the same as that of cellular and molecular components.

\section{TOP DOWN APPROACH:}

NANOCOMPOSITES: Nanohybrid and nanofilled RBCs are generally the two types of composite restorative materials, characterized by filler-particle sizes of $\leq 100$ $\mathrm{nm}$, referred to under the term "nanocomposite" ". They have desirable properties like High surface area to volume ratio, High aspect ratio, Adding carbon nanotubes improves electrical and thermal conductivity, Enhanced optical, dielectric properties, heat resistance and mechanical properties,

Advantages over conventional composites include: Superior flexural strength, modulus of elasticity, and translucency, Superior polish and gloss comparable to microfill composites, $50 \%$ reduction in filling shrinkage and Excellent handling properties.

\section{NANO-COMPOSITE DENTURE TEETH:}

Nanocomposite denture teeth are made of Polymethylmethacrylate (PMMA) and homogeneously distributed nanofillers. Its main advantages are excellent polishing ability and stain-resistant, superb esthetics, lively surface structure and enhanced wear resistance and surface hardness.

NANOTECHNOLOGY FOR GIC: It is a combination of fluoroaluminosilicate glass, nanofillers, and nanofiller clusters. It also shows high fluoride release that is rechargeable after being exposed to a topical fluoride source. In vitro tests showed that the nanoionomer (Ketac N100) has the ability to create a caries inhibition zone after acid exposure ${ }^{\text {. }}$.

\section{IMPROVING ENDODONTICS:}

Nanomaterial Enhanced Retrofil Polymer (NERP) is a nano root end filling material. It prevents agglomeration, have high dentin bond strength, reinforce the surface hardness of exposed dentin, penetrate dentinal tubules of diameter $5-10 \mathrm{~nm}$ to provide additional nano retention ${ }^{10}$.

IMPRESSION MATERIALS: Nanofillers are integrated in vinylpolysiloxanes, producing a unique edition of siloxane impression material. The presence of the nanostructure increases the fluidity of the material, especially when pressure is applied $^{10}$.

NANOTITANIUM IMPLANTS: Patients experiences shorter post surgery healing time and a more reliable integration of these new implants into their body. It is highly compatible with bone. Provide stronger, up to 20 times faster bonding with improved strength, long life and improved wear and tear ${ }^{11}$.

NANONEEDLES: Combination of properties of ordinary austenitic stainless and low alloyed ferritic steels. Vaccines may be made more comfortable, by using nanometer-scale needles, to which human nerves are insensitive, rather than a painful injection $^{11}$. 
BONE REPLACING MATERIAL: The hydroxyapatite nanoparticles have nanocrystallites that show a loose microstructure in which nanopores are situated between the crystallites. The surface of the pores is modified in such a manner, that it literally "hangs on" to the proteins. Most bone replacement material mainly acts on a surface through these pores on which proteins can configure. That's why the cells recognize it as body's own material ${ }^{10}$.

\section{BIOMIMETIC APPROACH}

DRUG FORMULATIONS: Nanoparticles can be used in targeted drug delivery at the site of disease to improve the uptake of poorly soluble drugs and the targeting of drugs to a specific site.

\section{DIAGNOSTIC APPLICATIONS:}

Nanotechnology also plays a key role in diagnostic applications by providing sensors for medical and environmental monitoring and Lab-on-a-chip diagnostic techniques.

\section{Future Research \& Challenges In Nanotechnology}

\section{Future Applications Of Nanotechnology}

- Longer-lasting medical implants.

- The capability to map an individual's entire genetic code almost instantaneously.

- The ability to extend life by $50 \%$ from present expectations.

- Light and strong materials for defence, aerospace, automotive, and medical applications.

\section{Problems \& Challenges In Nanotechnology}

Nanotechnology faces many challenges that need to be overcome such as - precise positioning and assembly of molecular scale part, economical nanorobot mass production technique, biocompatibility, simultaneous coordination of activities of large numbers of independent micron scale robot and social issues of public acceptance, ethics and regulation and human safety ${ }^{6}$.The risk to health and environment from nanoparticles and nanomaterials and the risks posed by molecular manufacturing and social risks need further investigations.

\section{CONCLUSION}

Nanotechnology offers great potential in the field of dentistry ranging from dental restorative materials to implants to surgical procedures to bone replacement material etc. However, with every great good, comes great evil. While it is appropriate to examine carefully the risks and potential toxicity of nanoparticles and other products of nanoscale technology, the greatest risks are posed by malicious and unwise use of molecular manufacturing. However, it can be predicted that nanotechnology will have a great impact on dental research, dental prevention, diagnostics and treatment solutions.

\section{Acknowledgement: None}

\section{Conflict of Interest: None}

\section{Source of Funding: None}

\section{REFERENCES}

1. Gribbin, John and Gribbin, Mary, 1997. Richard Feynman.ALife in Science. Dutton. p. 170.

2. Das S, Gates AJ, Abdu HA, Rose GS, Picconatto CA and Ellenbogen JC., 2007. "Designs for Ultra-Tiny,Special-Purpose Nanoelectronic Circuits". IEEE Transactions on Circuits and Systems I (11):2528-2540

3. Ghalanbor Z, Marashi SA and Ranjbar B, 2005. "Nanotechnology helps medicine: nanoscale swimmers and their future applications". Med Hypotheses (1): 198 199.

4. Freitas RA Jr. Nanodentistry. J Am Dent Assoc. 2000 Nov;131(11):1559-65. doi: 10.14219/jada.archive.2000.0084. PMID: 11103574 .

5. Nagpal Archana, Kaur Jasjit, Sharma Shuchita, BansalAarti and Sachdev Priyanka, 2011. Nanotechnology The Era of 
Molecular Dentistry. Indian J. Dent. Sci. 3 (5).

6. Shetty Neetha J., Swati P. and David K., 2013. Nanorobots: Future in dentistry The Saudi Dental Journal :49-52

7. Patil Mallanagouda, Singh Mehta Dhoom and Gurva Sowjanya 2008. Future Impact of Nanotechnology on Medicine and Dentistry. Jour of Indian Society of Periodontology, (2); 34-40.

8. Mitra Sumita B., Wu Dong, and Holmes Brian N, 2003. An application of Nanotechnology in Advanced Dental Materials JADA (10): 1382-1390

9. Uysal Tancan, Yagci Ahmet, Uysal Banu and Akdogan Gülsen, 2010. Are nano- composites and nanoionomers suitable for orthodontic bracket. European Journal of Orthodontics ;78-82.

10. Albrektsson T., Sennerby L. and Wennerberg A., 2008. State of the Art of Oral Implants. Periodontology , 1526

11. Kumar Saravana R and Vijayalakshmi R, 2006. Nanotechnology in Dentistry. Ind J Dent Res; (2):62 -65

How to cite this article: Amina, Ahmed S, Rajput G et.al. Nanotechnology - a panoramic outlook of its applications and future in dentistry. Int J Health Sci Res. 2022; 12(2):176180. DOI: https://doi.org/10.52403/ijhsr. 20220224 